\title{
EFEITO DA ÉPOCA, DO NÚMERO DE APLICAÇÕES E DE DOSES DE FUNGICIDA NO CONTROLE DA GIBERELA EM TRIGO*
}

\author{
EDIVAN PANISSON**, ERLEI M. REIS \& WALTER BOLLER
}

Faculdade de Agronomia e Medicina Veterinária, Mestrado em Agronomia, Área de Concentração Fitopatologia, Universidade de Passo Fundo, CEP 99001-970, Cx. Postal 601, Passo Fundo, RS, e-mail: erleireis@uol.com.br

(Aceito para publicação em 04/06/2002)

Autor para correspondência: Erlei Melo Reis

PANISSON, E., REIS, E.M. \& BOLLER, W. Efeito da época, do número de aplicações e de doses de fungicida no controle da giberela em trigo. Fitopatologia Brasileira 27:495-499. 2002.

\section{RESUMO}

A giberela é considerada uma doença de infecção floral. Para seu desenvolvimento, são necessárias temperaturas superiores a $20^{\circ} \mathrm{C}$ e períodos de molhamento das espigas maiores do que $72 \mathrm{~h}$ durante a antese do trigo (Triticum aestivum). Os objetivos do presente trabalho foram estudar o momento ideal durante a antese para realizar a aplicação de fungicida, o efeito do número de aplicações e de doses de fungicida sobre o controle da doença e sobre a qualidade dos grãos colhidos. Até sete aplicações realizadas durante a antese não erradicaram a doença (controle de 91\%). Duas e três aplicações realizadas durante a antese apresentaram controle superior em relação a uma única aplicação. O controle médio dos tratamentos foi de $67 \%$ em relação a severidade. O incremento de grãos obtido de $32 \%$, justifica economicamente a aplicação de fungicidas para o controle da doença em plena floração.

Palavras-chave adicionais: controle químico, Fusarium graminearum e Gibberella zeae.

\section{ABSTRACT \\ Timing, number of applications, and rates of fungicide to control head blight in wheat}

Head blight is one of the most damaging wheat (Triticum aestivum) diseases, causing reduction of grain yield, grain quality, flour protein levels, seed germination and vigor, and production of micotoxins. Head blight is considered a floral disease because the fungus enters the host plant through the anthers during anthesis. Occurrence of head blight is conditioned by temperatures above $20{ }^{\circ} \mathrm{C}$ and wetness periods longer than $72 \mathrm{~h}$. This research aimed at studying the timing during anthesis for fungicidal sprays, the effect of rates of fungicide and number of sprays on head blight control and on grain yield and quality. Uneven anthesis is the main cause for the low efficacy of fungicides in the field. Even six and seven sprays during anthesis did not eradicate the disease (maximum control of 91\%). Two and three applications improved the disease control over one spray. On average, the fungicidal sprays controlled head blight by $67 \%$ and increased grain yield by $32 \%$. These results showed that chemical control of head blight is useful and would be practical at full anthesis, with no rate reduction recommended.

\section{INTRODUÇÃO}

A giberela é causada pelo fungo Gibberella zeae (Schw.) Petch, anamórfo Fusarium graminearum (Schwabe). Nos últimos anos a doença tem aumentado de intensidade, ocorrendo em quase todas as regiões onde se cultiva o trigo (Triticum aestivum L.) (Parry et al., 1995; McMullen et al., 1997). Uma provável causa para o aumento da intensidade da doença pode estar relacionada com a maior quantidade de inóculo presente no ar, devido à manutenção dos restos culturais sobre a superfície do solo no sistema plantio direto (Mauler-Machnik \& Zahn,1994; Panisson et al., 2001).

No Brasil, são poucos os trabalhos que estimaram, com confiabilidade, os danos causados pela doença. Reis et al. (1996a) quantificando os danos causados pela doença no

\footnotetext{
* Parte da Dissertação de Mestrado do primeiro autor. Universidade de Passo Fundo.

** Bolsista da CAPES
}

período de 1984 a 1994, observaram um dano médio de 5,4\%. Recentemente, Panisson et al. (2001) quantificaram danos em trigo de até $25,9 \%$.

Das doenças do trigo, a giberela é a que apresenta as maiores dificuldades de controle devido: a) esporadicidade de sua ocorrência (Reis, 1988); b) momento oportuno para realizar o controle (Reis, 1988; Mauler-Machnik \& Zahn, 1994; Parry et al., 1995; Mesterházy \& Bartók, 1996; McMullen, et al., 1997); c) dificuldade do fungicida em atingir os sítios de infecção (Reis et al., 1996b); d) baixa eficiência de controle dos fungicidas disponíveis (Parry et al., 1995; Mesterházy \& Bartók, 1996).

Em cereais de inverno, entende-se por floração o período em que os órgãos masculinos da flor (anteras) estão expostos sendo sinônimo de antese (Zadocks, et al., 1974). As anteras soltas são aquelas que ficam totalmente expostas, pendentes externamente pelo filete; anteras presas são as que permanecem aprisionadas entre as extremidades superiores das glumas somente com o ápice visível. A deiscência ocorre 
apenas com as anteras soltas (Reis, et al., 1996); algumas anteras podem permanecer no interior da flor não enquadrando-se como presas ou soltas.

Arthur (1891), ao descrever a doença, observou que se tratava de uma doença de infecção floral. Este fato foi confirmado, mais tarde, por diversos pesquisadores (Andersen, 1948; Strange \& Smith, 1971; Strange et al., 1974; Reis, 1988). Reis et al. (1996b) descrevem que para se obter sucesso no controle da doença os fungicidas devem ser aplicados de modo a atingir o maior número de anteras. Moschini \& Fortugno (1996) salientam que a recomendação de fungicidas para o controle da giberela pode ser baseada nas previsões climáticas ocorrentes durante a antese, ou seja, se as condições para ocorrência forem favoráveis, a aplicação deve ser realizada.

Reis et al. (1996b), estudando a eficiência de diferentes fungicidas, em casa de vegetação, comprovaram que quando os fungicidas foram aplicados de forma a cobrir as anteras apresentaram uma eficiência de controle de até $93 \%$. Salientam que, no campo esta eficiência é menor, principalmente, pela dificuldade de deposição do fungicida sobre as anteras (Reis, 1988).

Os objetivos do presente trabalho foram determinar: a) a época ideal durante a antese para realizar a aplicação de fungicidas; b) o efeito do número de aplicações neste período; c) o efeito do fracionamento da dose do fungicida sobre o controle da doença e d) o efeito dos tratamentos com fungicida sobre o rendimento e a qualidade dos grãos colhidos.

\section{MATERIAL E MÉTODOS}

O experimento foi conduzido no Centro de Extensão e Pesquisa Agronômica da Faculdade de Agronomia e Medicina Veterinária da Universidade de Passo Fundo, em Passo Fundo, RS, na safra 2000. A infecção ocorreu sob condições naturais. Em alguns períodos, durante a antese, foi utilizada irrigação por aspersão para garantir o molhamento das espigas e favorecer a infecção.

Utilizou-se o trigo cultivar BR 23, semeada em duas épocas 26 de junho e 8 de julho de 2000, respectivamente. Os tratos culturais foram realizados de acordo com as recomendações técnicas da cultura (Recomendações..., 2000). Nas duas épocas de semeadura foi realizada uma aplicação de fungicida triadimenol (125 g.i.a.ha $\left.{ }^{-1}\right)$, no dia 7 de setembro para o controle de oídio (Blumeria graminis Speer f. sp. tritici Em. Marchal) em todas as parcelas, exceto no Tratamento 1.

$O$ fungicida usado no controle da giberela foi o tebuconazole $200 \mathrm{CE}$. Os tratamentos foram: (1) Testemunha sem aplicação de fungicida durante todo o ciclo; (2) Testemunha somente com a aplicação de fungicida para o controle de doenças foliares; (3) Uma aplicação no estádio 60 (início da antese) (150 g.i.a.ha $\left.{ }^{-1}\right)$; (4) Uma aplicação no estádio 65 (metade da antese) (150 g.i.a.ha-1 $)$; (5) Duas aplicações nos estádios 60 e 65 (75 g.i.a.ha ${ }^{-1}+75$ g.i.a.ha $\left.{ }^{-1}\right)$; (6) Duas aplicações nos estádios 60 e 65 (150 g.i.a.ha-1 +150 g.i.a.ha-1); (7) Três aplicações nos estádios 60, 65 e 68 (final da antese) (50 g.i.a.ha ${ }^{-1}+50$ g.i.a.ha ${ }^{-1}+50$ g.i.a.ha $\left.{ }^{-1}\right) ;(8)$ Três aplicações nos estádios 60, 65 e 68 (150 g de i.a./ha + 150 g.i.a.ha ${ }^{-1}+150$ g.i.a.ha ${ }^{-1}$ ); e (9) Parcela controle (sete e seis aplicações de 150 g.i.a.ha ${ }^{-1}$ durante a antese, na primeira e segunda época, respectivamente).

As aplicações foram realizadas com pulverizador costal pressurizado com $\mathrm{CO}_{2}$, equipado com uma barra com três bicos com pontas duplo leque (TJ60 11002). A pressão de trabalho foi de $200 \mathrm{kPa}$, que com deslocamento de $1 \mathrm{~m} . \mathrm{s}^{-1}$ determinava um volume de calda de 2001. ha $^{-1}$. A aplicação foi realizada deslocando-se pelas laterais da parcela, para não causar amassamento ou remover as gotas da calda depositadas nas anteras. $\mathrm{O}$ tamanho das parcelas foi de $6 \mathrm{~m} \times 2,55 \mathrm{~m}$.

Durante a aplicação monitorou-se as condições ambientais com um termo-higro-anemômetro (Kestrel 3000). Os dados da temperatura, da umidade relativa e da velocidade do vento no momento da aplicação estão na Tabela 1.

Determinou-se a quantidade de espigas com anteras em $1,0 \mathrm{~m}^{2} /$ parcela e a proporção de anteras soltas e presas em 50 espigas. Avaliou-se a incidência, a porcentagem de espiguetas gibereladas, a severidade, o rendimento de grãos, o peso hectolítrico, o peso de mil sementes e a incidência de F. graminearum nos grãos colhidos. As três primeiras variáveis foram determinadas no estádio 85 da escala fenológica de Zadocks et al., (1974). A incidência foi determinada em $0,65 \mathrm{~m}^{2}$, considerando-se espiga doente a que apresentasse no mínimo duas espiguetas com sintomas. A porcentagem de espiguetas gibereladas foi determinada em 25 espigas coletadas ao acaso e a severidade foi calculada através do produto da incidência pela porcentagem de espiguetas gibereladas dividindo-se por 100. A colheita foi realizada em $5,1 \mathrm{~m}^{2}$. No cálculo do rendimento de grãos e do peso de mil sementes a umidade foi convertida para $13 \%$. O rendimento relativo foi calculado através da porcentagem de incremento de grãos em relação às testemunhas. Determinouse em 50 sementes por parcela a incidência de $F$. graminearum. A assepsia das sementes foi realizada com hipoclorito de sódio a $50 \%$ (produto comercial), durante $3 \mathrm{~min}$, plaqueadas em meio seletivo a Fusarium spp. (Nash \& Snyder, 1962) e incubadas em câmara climatizada a uma temperatura de $25 \pm 2{ }^{\circ} \mathrm{C}$, sob luz branca com fotoperíodo de $12 \mathrm{~h}$. No sétimo dia avaliou-se a porcentagem de sementes infetadas.

O delineamento experimental utilizado foi de blocos casualizados com quatro repetições por tratamento. As médias

TABELA 1 - Condições ambientais durante a aplicação dos fungicidas. Passo Fundo, RS, 2000

\begin{tabular}{ccccc}
\hline \hline \multirow{2}{*}{$\begin{array}{c}\text { Época/ } \\
\text { Estádio* }\end{array}$} & $\begin{array}{c}\text { Data de } \\
\text { aplicação }\end{array}$ & T ( ${ }^{\mathbf{} C)}$ & UR (\%) & $\begin{array}{c}\text { Vento } \\
\text { (km/h) }\end{array}$ \\
\cline { 3 - 5 } & $30 / 09$ & 24 & 69 & 7,2 \\
$1 / 60$ & $03 / 10$ & 25 & 72 & 10 \\
$1 / 65$ & $06 / 10$ & 13 & 59 & 3 \\
\hline $2 / 68$ & $06 / 10$ & 15 & 54 & 3 \\
$2 / 65$ & $10 / 10$ & 18 & 90 & 4 \\
$2 / 68$ & $14 / 10$ & 17 & 90 & 3 \\
\hline
\end{tabular}


Efeito da época, do número de aplicações e de doses...

obtidas foram comparadas pelo teste de Duncan a $5 \%$ de probabilidade. $\mathrm{Na}$ análise da variância da eficiência de controle, os dados foram transformados para arco seno $\sqrt{x}+0,5$.

\section{RESULTADOS E DISCUSSÃO}

A quantidade de anteras nas infrutescências do trigo foi muito variável, verificado pelo alto desvio padrão observado, principalmente nas anteras presas (Tabela 2). Nas duas épocas avaliadas, verificou-se pequeno número de espigas com anteras no início da antese no momento da aplicação do fungicida. Este fato poderia acarretar uma menor eficácia do fungicida, já que poucas anteras receberiam o fungicida. A desuniformidade da antese em trigo foi analisada por Reis (1988) que observou que a duração da mesma pode ser de quatro dias em uma espiga, de 12 dias em uma planta e de até 25 dias em uma lavoura. A desuniformidade da floração pode ser um dos motivos do baixo nível de controle obtidos com os fungicidas no campo. $\mathrm{O}$ número de anteras presas aumentou com o passar do tempo. Aplicações de fungicida no final da antese poderiam atingir maior número

TABELA 2 - Estádios, quantidade de espigas com anteras e número de anteras por espiga de trigo (Triticum aestivum). Passo Fundo, RS, 2000

\begin{tabular}{cccc}
\hline \hline Época/ & Espigas com & \multicolumn{2}{c}{ Quantidade de anteras** } \\
\cline { 3 - 4 } Estádio* & anteras (\%) & Solta & Presa \\
\hline $1 / 60$ & 14 & $35,9 \pm 16,0$ & $1,9 \pm 1,8$ \\
$1 / 65$ & 85 & $45,9 \pm 18,8$ & $5,3 \pm 3,5$ \\
$1 / 68$ & 100 & $5,1 \pm 6,8$ & $5,6 \pm 3,8$ \\
\hline $2 / 60$ & 19 & $34,1 \pm 12,8$ & $5,5 \pm 4,9$ \\
$2 / 65$ & 54 & $36,6 \pm 14,8$ & $6,4 \pm 4,4$ \\
$2 / 68$ & 100 & $3,5 \pm 5,0$ & $7,6 \pm 3,9$ \\
\hline
\end{tabular}

* Estádio da escala fenológica de Zadoks et al. (1974).

** Número médio \pm desvio padrão. de anteras presas prevenindo infecções pós-antese. Por outro lado, de nada adiantaria proceder a aplicação neste estádio, pois os fungicidas recomendados apresentam apenas efeito protetor (Reis, 1988). Neste momento a infecção através das anteras soltas já poderia ter ocorrido. Milus \& Parsons (1994) verificaram que os fungicidas quando aplicados antes da antese não têm efeito sobre a doença. Embora a antese seja desuniforme, a aplicação dos fungicidas deve ser realizada durante este estádio (Reis, 1988; Mauler-Machnik \& Zahn, 1994; Parry et al., 1995; Mesterházy \& Bartók, 1996; Reis et al., 1997; McMullen, 1998) pois a calda do fungicida necessariamente deve atingir as anteras para que possa haver controle (Reis, et al., 1996). A seleção de cultivares com período curto e uniforme de antese poderia favorecer o controle químico além de ser uma forma de escape à infecção pelo patógeno.

Na primeira época, observou-se que a aplicação dos fungicidas no início ou em antese plena (Tratamentos 3 e 4) não apresentou diferença entre si, em nenhuma variável avaliada (Tabela 3). Quando se realizaram duas aplicações (Tratamentos 5 e 7) foi reduzida a severidade em relação a uma única aplicação. Quando se utilizaram três aplicações (Tratamentos 6 e 8) foi observada menor intensidade da doença, com reflexo em um maior incremento do rendimento de grãos (Tabela 4).

O rendimento de grãos foi a variável que mais respondeu aos tratamentos (Tabela 4). Houve resposta significativa de todos os tratamentos aplicados durante a antese. $\mathrm{O}$ incremento no rendimento de grãos foi em média $27,8 \%$ e $36,6 \%$ em relação a testemunha (Tratamento 1 ), para a primeira e segunda épocas, respectivamente, confirmando os relatos de Martin \& Johnston (1982) e Wong et al. (1992) os quais verificaram que o maior efeito da aplicação do fungicida foi sobre o rendimento de grãos.

A incidência média de $80,4 \%$ e de $64,2 \%$ de $F$.

TABELA 3 - Efeito do momento, número de aplicações e doses do fungicida tebuconazole sobre a incidência (I), o número de espiguetas gibereladas (NEG) e a severidade (S) em trigo (Triticum aestivum). Passo Fundo, RS, 2000

\begin{tabular}{|c|c|c|c|c|c|c|}
\hline \multirow{2}{*}{$\begin{array}{l}\text { Tratamento } \\
\text { Estádio*/dose** }\end{array}$} & \multicolumn{3}{|c|}{ Época 1} & \multicolumn{3}{|c|}{ Época 2} \\
\hline & I (\%) & NEG (\%) & S (\%) & I (\%) & NEG (\%) & S (\%) \\
\hline $1-$ Testemunha 1 & $73,3 \mathrm{a}$ & $52,9 \mathrm{a}$ & $38,8 \mathrm{a}$ & $70,2 \mathrm{a}$ & $49,4 \mathrm{a}$ & $34,9 \mathrm{a}$ \\
\hline $3-60 / 150$ & 44,6 cde & $38,0 \mathrm{~cd}$ & 16,6 cde & $45,2 \mathrm{bc}$ & $38,4 \mathrm{~b}$ & $17,5 \mathrm{~b}$ \\
\hline $4-65 / 150$ & $48,3 \mathrm{~cd}$ & $39,6 \mathrm{bc}$ & $18,9 \mathrm{~cd}$ & $42,5 \mathrm{bc}$ & $31,0 \mathrm{bc}$ & $12,8 \mathrm{bc}$ \\
\hline $5-60$ e $65 / 75+75$ & $54,8 \mathrm{bc}$ & $35,7 \mathrm{~cd}$ & $19,5 \mathrm{c}$ & $42,4 \mathrm{bc}$ & $36,3 \mathrm{bc}$ & $15,3 \mathrm{~b}$ \\
\hline $6-60,65$ e $68 / 50+50+50$ & $36,3 \mathrm{e}$ & $30,6 \mathrm{~cd}$ & $11,4 \mathrm{e}$ & $44,8 \mathrm{bc}$ & $34,0 \mathrm{bc}$ & $15,7 \mathrm{~b}$ \\
\hline $7-60$ e $65 / 150+150$ & $42,9 \mathrm{de}$ & $32,4 \mathrm{~cd}$ & $13,9 \mathrm{cde}$ & $31,8 \mathrm{~cd}$ & $28,8 \mathrm{bcd}$ & 9,4 bcd \\
\hline $8-60,65$ e $68 / 150+150+150$ & $34,2 \mathrm{e}$ & $34,5 \mathrm{~cd}$ & $12,4 \mathrm{de}$ & $20,7 \mathrm{~d}$ & $27,3 \mathrm{~cd}$ & $5,9 \mathrm{~cd}$ \\
\hline 9 - Parcela controle & $12,8 \mathrm{f}$ & $27,3 \mathrm{~d}$ & $3,5 \mathrm{f}$ & $15,1 \mathrm{~d}$ & $20,4 \mathrm{~d}$ & $3,1 \mathrm{~d}$ \\
\hline Média & 45,6 & 37,7 & 18,4 & 40,7 & 35,1 & 15,74 \\
\hline CV (\%) & 16,1 & 18,7 & 22,6 & 28,6 & 18,9 & 34,6 \\
\hline
\end{tabular}

* Estádio da escala fenológica de Zadoks et al. (1974).

** Dose do fungicida tebuconazole (g.i.a.ha ${ }^{-1}$ )

Médias seguidas da mesma letra na coluna não diferem significativamente pelo teste de Duncan (5\%). 
TABELA 4 - Efeito do momento, número de aplicações e doses do fungicida tebuconazole sobre o rendimento de grãos (RG), o rendimento relativo (RR), o peso hectolítrico (PH), o peso de 1.000 sementes (PMS) e incidência de Fusarium graminearum nas sementes colhidas (IS) em trigo (Triticum aestivum). Passo Fundo, RS, 2000

\begin{tabular}{|c|c|c|c|c|c|c|c|c|c|c|c|c|}
\hline \multirow[b]{2}{*}{ Estádio*/dose** } & \multicolumn{6}{|c|}{ Época 1} & \multicolumn{6}{|c|}{ Época 2} \\
\hline & $\begin{array}{c}\text { RG } \\
\text { kg.ha }{ }^{-1}\end{array}$ & $\begin{array}{c}\mathbf{R R}^{1} \\
\% \\
\end{array}$ & $\begin{array}{c}\mathbf{R R}^{2} \\
\% \\
\end{array}$ & PH & $\begin{array}{c}\text { PMS } \\
\mathrm{g}\end{array}$ & $\begin{array}{l}\text { IS } \\
\% \\
\end{array}$ & $\begin{array}{c}\text { RG } \\
\text { kg.ha } \\
\end{array}$ & $\begin{array}{c}\mathbf{R R}^{\mathbf{1}} \\
\% \\
\end{array}$ & $\begin{array}{c}\mathbf{R R}^{\mathbf{2}} \\
\% \\
\end{array}$ & PH & $\begin{array}{c}\text { PMS } \\
\mathrm{g}\end{array}$ & $\begin{array}{l}\text { IS } \\
\% \\
\end{array}$ \\
\hline $1-$ Testemunha 1 & $2838 \mathrm{f}$ & - & - & $74,3 \mathrm{~g}$ & $30,6 \mathrm{c}$ & $90,6 \mathrm{a}$ & $3085 \mathrm{~d}$ & - & - & $73,5 \mathrm{c}$ & $34,9 \mathrm{c}$ & $80,7 \mathrm{a}$ \\
\hline 2 - Testemunha 2 & 3149 e & 11,0 & - & $74,6 \mathrm{f}$ & $29,3 \mathrm{bc}$ & $89,6 \mathrm{ab}$ & $3280 \mathrm{~d}$ & 6,3 & - & $73,9 \mathrm{c}$ & $35,3 \mathrm{c}$ & $72,4 \mathrm{ab}$ \\
\hline $3-60 / 150$ & $3366 \mathrm{de}$ & 18,6 & 6,9 & 76,3 ed & $30,6 \mathrm{bc}$ & $83,9 \mathrm{abc}$ & $3988 \mathrm{c}$ & 29,3 & 21,6 & $75,4 \mathrm{~b}$ & $37,1 \mathrm{ab}$ & $67,2 \mathrm{abc}$ \\
\hline $4-65 / 150$ & $3484 \mathrm{~cd}$ & 22,8 & 10,6 & 75,6 ef & $31,0 \mathrm{bc}$ & $81,8 \mathrm{abc}$ & $3942 \mathrm{c}$ & 27,8 & 20,2 & $75,0 \mathrm{~b}$ & $37,5 \mathrm{ab}$ & $64,1 \mathrm{bc}$ \\
\hline $5-60$ e $65 / 75+75$ & $3600 \mathrm{bcd}$ & 26,8 & 14,3 & 77,3 bcd & $32,4 \mathrm{~b}$ & $75,5 \mathrm{c}$ & $4250 \mathrm{c}$ & 37,8 & 29,6 & $75,0 \mathrm{~b}$ & 36,6 bc & $71,9 \mathrm{ab}$ \\
\hline $6-60,65$ e $68 / 50+50+50$ & $3564 \mathrm{bcd}$ & 25,6 & 13,2 & $76,4 \mathrm{bcd}$ & $31,3 \mathrm{bc}$ & $81,3 \mathrm{abc}$ & $4176 \mathrm{c}$ & 35,4 & 27,3 & $75,7 \mathrm{~b}$ & $37,6 \mathrm{ab}$ & $63,0 \mathrm{bc}$ \\
\hline $7-60$ e $65 / 150+150$ & $3735 \mathrm{bc}$ & 31,6 & 18,6 & $77,5 \mathrm{bc}$ & $33,0 \mathrm{~b}$ & $78,1 \mathrm{c}$ & $4333 \mathrm{bc}$ & 40,5 & 32,1 & $75,5 \mathrm{~b}$ & $37,4 \mathrm{ab}$ & $67,2 \mathrm{abc}$ \\
\hline $8-60,65$ e $68 / 150+150+150$ & $3802 \mathrm{~b}$ & 34,0 & 20,7 & $77,6 \mathrm{~b}$ & $33,0 \mathrm{~b}$ & $79,1 \mathrm{bc}$ & $4692 \mathrm{ab}$ & 52,1 & 43,0 & $77,1 \mathrm{a}$ & $38,0 \mathrm{ab}$ & $54,2 \mathrm{c}$ \\
\hline 9 - Parcela controle & $4312 \mathrm{a}$ & 51,9 & 36,9 & $79,8 \mathrm{a}$ & $35,6 \mathrm{a}$ & $64,1 \mathrm{c}$ & $5055 \mathrm{a}$ & 63,9 & 54,1 & 77,9 a & $38,6 \mathrm{a}$ & $37,0 \mathrm{~d}$ \\
\hline Média & 3539 & 27,8 & 17,3 & 76,6 & 31,85 & 80,4 & 4089 & 36,6 & 32,6 & 75,5 & 37,0 & 64,2 \\
\hline CV (\%) & 4,8 & - & - & 0,9 & 5,1 & 6,8 & 6,8 & - & - & 0,8 & 3,1 & 15,8 \\
\hline
\end{tabular}

* Estádio da escala fenológica de Zadoks et al. (1974).

** Dose do fungicida tebuconazole (g.i.a.ha ${ }^{-1}$ )

Médias seguidas da mesma letra na coluna não diferem significativamente pelo teste de Duncan (5\%); n.s. não significativo.

${ }^{1}$ Incremento de grãos em relação ao Tratamento 1.

${ }^{2}$ Incremento de grãos em relação ao Tratamento 2.

graminearum observada nas sementes, na primeira e segunda épocas, respectivamente, indicam que o tratamento com fungicida teve pouco efeito sobre a infecção dos grãos.

$\mathrm{Na}$ parcela controle, observou-se controle mais eficiente, propiciando aumento significativo de todas as variáveis avaliadas. É importante destacar que mesmo com seis ou sete aplicações durante a antese (intervalo de 15 dias) não foi possível erradicar a doença. Resultados obtidos a campo relatando controle superior a 91\% (Figura 1), como resultado de uma única aplicação, devem ser observados com cautela, uma vez que pode ter ocorrido problemas de amostragem e/ou metodologia de avaliação. Pode-se deduzir que o potencial de controle dos fungicidas em aplicações convencionais no campo deve ser em torno de 60-70\%. A baixa eficiência de controle no campo é relatada por diversos pesquisadores (Martin \& Johnston, 1982; Milus \& Weight, 1998; McMullen, 1998; Schaafsma et al., 1998). Uma eficiência maior talvez possa ser obtida utilizando-se cultivares com floração uniforme. Os fungicidas atualmente usados parecem ter pouco efeito em restringir o crescimento micelial do fungo na colonização da espiga, atuando basicamente como protetores da infecção inicial nas anteras (Tabela 4).

Deste trabalho pode-se inferir que o melhor critério para avaliar o controle da doença foi a severidade que reflete a intensidade da doença visualizada no campo. Considerandose o critério da incidência e do número de espiguetas gibereladas, o controle foi inferior ao encontrado com a severidade.

$\mathrm{Na}$ China, nas regiões onde existem maiores probabilidades de ocorrer epidemias são realizadas duas aplicações de fungicidas benzimidazóis na antese, uma no início e outra sete dias após (Parry et al., 1995). Reis (1988) também relata
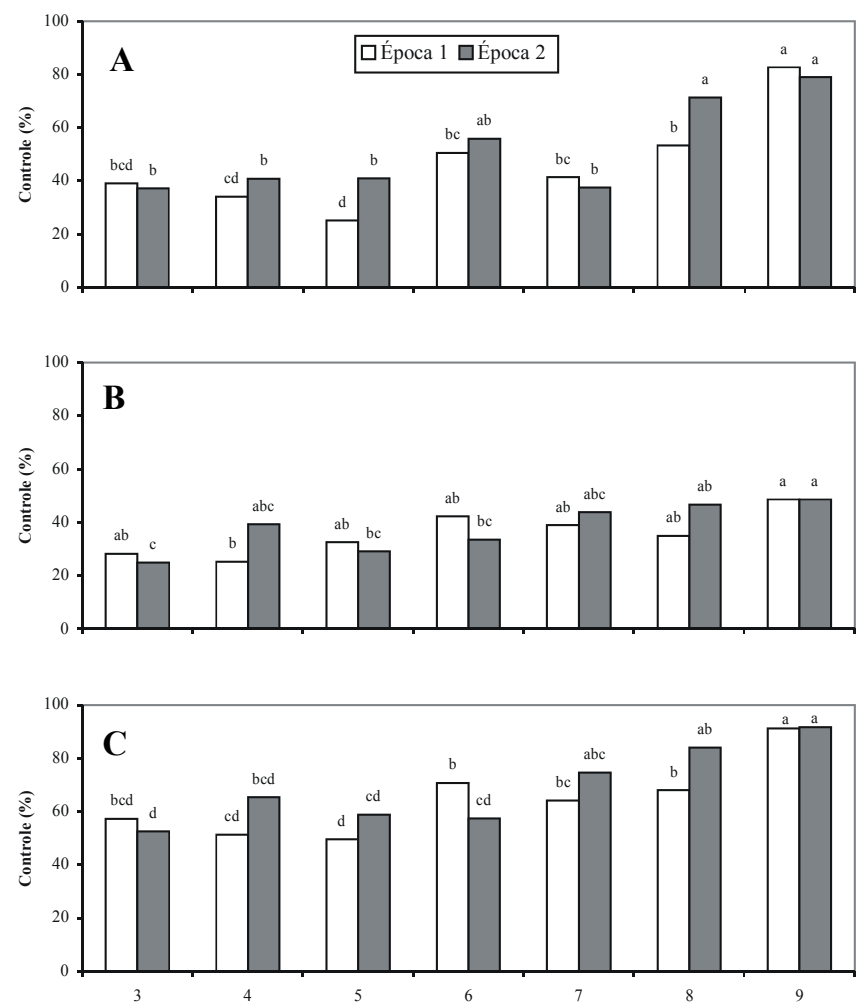

FIG. 1 - Efeito da aplicação do fungicida tebuconazole aplicados em diferentes momentos, número de aplicações e doses sobre no controle de giberela em trigo (Triticum aestivum), A) controle da incidência; B) controle do número de espiguetas gibereladas; C) controle da severidade. Passo Fundo, RS, 2000. Colunas com a mesma letra, na mesma época, não diferem significativamente entre si, pelo teste de Duncan (5\%). 
Efeito da época, do número de aplicações e de doses...

que duas aplicações durante a antese poderiam ter melhor efeito. Pelos resultados observados nas duas épocas, verificouse que duas aplicações durante a antese, embora tenham aumentado a eficiência do fungicida, não tiveram efeito significativo em relação ao controle da doença, incremento no rendimento de grãos e sobre a qualidade dos mesmos, contrariando os relatos de Suty \& Mauler-Machnik (1996). Quando se realizam três aplicações durante a antese, o desempenho do fungicida foi superior, porém o efeito alcançado deve ser justificado economicamente.

Nas condições observadas no trabalho, uma única aplicação em floração plena, embora proporcione um controle inferior a $60 \%$, determinou um incremento no rendimento de grãos, no peso do hectolitro e no peso de mil sementes.

\section{AGRADECIMENTOS}

Os autores agradecem a CAPES e a Bayer S.A. o apoio financeiro na realização deste trabalho.

\section{REFERÊNCIAS BIBLIOGRÁFICAS}

ANDERSEN, A.L. The development of Gibberella zeae headblight of wheat. Phytopathology 38:595-611. 1948.

ARTHUR, J.C. Wheat Scab. Indiana Agricultural Experimental 36:192-232. 1891.

MARTIN, R.A. \& JOHNSTON, H.W. Effects and control of fusarium diseases of cereal grains in the Atlantic Provinces. Canadian Journal of Plant Pathology 4:210-216. 1982.

MAULER-MACHNIK, A. \& ZAHN, K. Ear fusarioses in wheat new findings on their epidemiology and control with Folicur (tebuconazole). Pflanzenschutz-Nachrichten Bayer 47:129-155. 1994.

McMULLEN, M., JONES, R. \& GALLENBERG, D. Scab of wheat and barley: a re-emerging disease of devastating impact. Plant Disease 81:1340-1348. 1997.

McMULLEN, M. Fungicide technology network of the national FHB initiative - 1988; report. In: Proceedings, the 1998 national fusarium head blight forum, Michigan, United States. 1998. pp.47-50.

MESTERHAZY, A. \& BARTOK, T. Control of Fusarium head blight of wheat by fungicides and its effect on the toxin contamination of the grains. Pflanzenschutz-Nachrichten Bayer 49:181-198. 1996.

MILUS, E.A. \& PARSONS, C.E. Evaluation of foliar fungicides for controlling Fusarium head blight of wheat. Plant Disease 78:697-699. 1994.

MILUS, E.A. \& WEIGHT, C.T. Efficacy of Quadris and Benlate applications on wheat scab in Arkansas. Proceedings, the 1998 national fusarium head blight forum, Michigan, United States. 1998. pp.51-52.

MOSCHINI, R.C. \& FORTUGNO, C. Predicting wheat head blight incidence using models based on meteorological factors in Pergamino, Argentina. European Journal of Plant Pathology 102:211-218.1996.

NASH, S.M. \& SNYDER, W.C. Quantitative estimatives by plate counts of propagules of the bean root rot Fusarium in field soil. Phytopathology 52:567-572. 1962.

PANISSON, E. Giberela em trigo: intensidade, danos e controle químico. (Dissertação Mestrado). Passo Fundo. Universidade de Passo Fundo. 2001.

PARRY, D.W., JENKINSON, P. \& McLEOD, L. Fusarium ear blight (scab) in small grain cereals - a review. Plant Pathology 44:207238. 1995.

Recomendações da Comissão Sul-Brasileira de pesquisa de trigo. Reunião da Comissão Sul-Brasileira de Pesquisa de Trigo, 32. Cruz Alta. 2000.

REIS, E.M. Doenças do Trigo III; Giberela. 2a ed. São Paulo. 1988.

REIS, E.M., BLUM, M.M.C., CASA, R.T. \& MEDEIROS, C.A. Grain losses caused by the infection of wheat heads by Gibberella zeae in southern Brazil, from 1984 to 1994. Summa Phytopathologica 22:134-137. 1996a.

REIS, E.M., BLUM, M.M.C. \& CASA, R.T. Controle químico de Gibberella zeae em trigo, um problema de deposição de fungicidas em anteras. Summa Phytopathologica 22:39-42. 1996b.

SCHAAFSMA, A.W., TAMBURIC-ILINCIC, L. \& MILLER, J.D. The effect of agronomic practice on the accumalation of deoxynivalenol (DON) in winter wheat fields in Ontario, 19961997. In: Proceedings, the 1998 national fusarium head blight forum, Michigan, Unites States. 1998. pp.7-10.

STRANGE, R.N. \& SMITH, H. A fungal growth stimulant in anthers which predisposes wheat to attack by Fusarium graminearum. Physiological Plant Pathology 1:141-150. 1971.

STRANGE, R.N., MAJER, J.R. \& SMITH H. The isolation and identification of choline and betaine as the two major components in anthers and wheat germ that stimulate Fusarium graminearum in vitro. Physiological Plant Pathology 4:277-290. 1974.

SUTY, A. \& MAULER-MACHNIK A. Fusarium head blight on wheat - new findings on the epidemiology and control of Gibberella zeae the teleomorph of Fusarium graminearum with Folicur. Pflanzenschutz-Nachrichten Bayer 49:55-70. 1996.

WONG, L.S.L., TEKAUZ, A., LEISLE, D., ABRAMSON, D. \& McKENZIE, R.I.H. Prevalence, distribution and importance of fusarium headblight in wheat in Manitoba. Canadian Journal of Plant Pathology 14:233-238. 1992.

ZADOCKS, J.C., CHANG, T.T. \& KONZAC, C.F. A decimal code for the growth stages of cereals. Weed Research 14:415-421. 1974 . 